
\title{
$\angle$ Research Square \\ Predicting ketosis during the transition period in Holstein Friesian cows using hematological and serum biochemical parameters on the calving date
}

\section{Seungmin $\mathrm{Ha}$}

National Institute of Animal Science, Rural Development Administration

\section{Seogjin Kang}

National Institute of Animal Science, Rural Development Administration

\section{Manhye Han}

National Institute of Animal Science, Rural Development Administration

Jihwan Lee

National Institute of Animal Science, Rural Development Administration

\section{Hakjae Chung}

National Institute of Animal Science, Rural Development Administration

\section{Sang-lk Oh}

National Institute of Animal Science, Rural Development Administration

\section{Suhee Kim}

Gyeongsang National University Hospital

Jinho Park ( $\sim$ jpark@jbnu.ac.kr)

Chonbuk National University

\section{Research Article}

Keywords: red blood cell (RBC) count, hemoglobin ( $\mathrm{Hb}), \mathrm{MCV}, \mathrm{MCH}, \mathrm{MCHC}$, RDW, and white blood cell (WBC), lymphocyte, monocyte

Posted Date: June 17th, 2021

DOl: https://doi.org/10.21203/rs.3.rs-605906/v1

License: (c) (i) This work is licensed under a Creative Commons Attribution 4.0 International License. Read Full License

Version of Record: A version of this preprint was published at Scientific Reports on January 17th, 2022. See the published version at https://doi.org/10.1038/s41598-022-04893-w. 


\section{Abstract}

Ketosis often occurs during the transition period in dairy cows, which leads to economic and welfare problems. Ketosis was reported to be associated with hematological and serum biochemical parameters. However, the association between the parameters on the calving date and ketosis during the postpartum transition period remains unclear. This study aimed to investigate the association. Blood samples were collected from the jugular vein of Holstein cows on calving date and $\beta$-hydroxybutyrate was tested once every three days (8 times in 21 days). The cows were divided into three groups: non-ketosis, subclinical ketosis, and clinical ketosis. The clinical ketosis group significantly had the highest values of mean corpuscular volume, mean corpuscular hemoglobin, $\beta$-hydroxybutyrate, non-esterified fatty acids, and total bilirubin, but the lowest values of red cell distribution width, the counts of white blood cell, monocyte, and eosinophil, albumin, alanine transaminase, lactate dehydrogenase, and amylase. Nonketosis group showed the opposite results $(p<0.05)$. The parameters are associated with the development and severity of ketosis. The findings suggest that these parameters on calving date may be useful indicators to identify dairy Holstein cow susceptible to ketosis during the transition period.

\section{Introduction}

Ketosis, which is associated with negative energy balance (NEB), is classified into three types: type $\rrbracket$

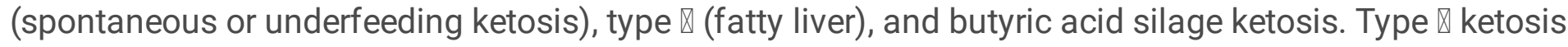
occurs in the postpartum transition period ${ }^{1,2}$. All dairy cows experience NEB, which is a higher energy requirement for milk production than energy intake through feed in early lactation ${ }^{3,4}$. However, NEB does not necessarily lead to ketosis in all dairy cows. The occurrence of ketosis depends on whether the cows overcome NEB through metabolic adaptation.

Ketosis causes economic and animal welfare concerns in the dairy farm industry. Many studies have reported factors associated with ketosis in dairy cows. including body condition score, breed, age at first calving, cow parity, calving season, dystocia, calving interval, dry period, prolonged previous lactation length, herd size, increased colostral production, milk protein percentage and 305-day milk yield and milk fat yield in the previous lactation, metritis, milk fever, retained placenta, and calf sex ${ }^{5-11}$. In addition, one previous study suggested that breeding values could be used to predict which Holstein cows are susceptible to ketosis ${ }^{12}$.

Hematological and serum biochemical parameters are widely used to evaluate and monitor health, and these parameters have been demonstrated to be associated with ketosis. For example, cows with severe ketosis displayed higher hematocrit and hemoglobin values, while mean corpuscular volume (MCV), mean corpuscular $(\mathrm{MCH})$, mean corpuscular hemoglobin concentration ( $\mathrm{MCHC})$, and red cell distribution width (RDW) did not show any difference between non-ketotic and ketotic cows ${ }^{13}$; in addition, cows with ketosis had significantly lower white blood cell, neutrophil, and eosinophil counts than those without ketosis ${ }^{14}$, and impaired white blood cell functions were associated with ketosis in dairy cows ${ }^{15}$; moreover, cows with ketosis showed increased non-esterified fatty acids (NEFA), aspartate 
aminotransferase, and total bilirubin (TB) and decreased levels of glucose, blood urea nitrogen (BUN), total protein, albumin (ALB), and triglyceride (TG) $2,16-21$; furthermore, concentrations of minerals, such as calcium, phosphorus, copper, and zinc, were associated with ketosis as well ${ }^{22-24}$. However, to the best of our knowledge, no previous studies have investigated the association of ketosis during the postpartum transition period with hematological and serum biochemical parameters on calving date.

We hypothesized that dairy Holstein cows show different levels of hematological and serum biochemical parameters on calving date before ketosis. Accordingly, the objectives of this study were 1) to identify which hematological and serum biochemical parameters are associated with ketosis and its severity (non-ketosis, subclinical ketosis, and clinical ketosis), 2) to investigate how the parameters is associated with ketosis, and 3) ultimately, to assess the ability of these parameters to predict ketosis during the postpartum transition period. The present findings would help to identify dairy cows that are at a high risk of ketosis and to establish a strategy to reduce ketosis damages.

\section{Results}

\subsection{Descriptive statistics for the dairy cows by ketosis}

Based on the highest $\beta$-hydroxybutyrate (BHBA) concentration in eight measurements during the postpartum transition period, 126 Holstein Friesian cows were divided into three groups: non-ketosis (NK), subclinical ketosis (SCK), and clinical ketosis (CK). The NK group accounted for $50 \%(n=63)$ of the population, while $26.98 \%(n=34)$ and $23.02 \%(n=29)$ of cows had SCK and CK, respectively. Both of SCK and CK groups occurred the most on day 9 . The parity and age were the highest in the CK group. Moreover, the CK group produced the most milk, while the NK group produced the least milk in the early transition period, even though the differences of daily milk yield during the entire transition period were less than $10 \%$ (Table 1 ). 
Table 1

Descriptive statistics for the Holstein Friesian cows included in the study. Abbreviation: NK, non-ketosis group; SCK, subclinical ketosis group; CK, clinical ketosis group. mean \pm standard of error of the means.

\begin{tabular}{|c|c|c|c|}
\hline & NK & SCK & CK \\
\hline Number & 63 & 34 & 29 \\
\hline Cow parity & $2.0 \pm 0.1$ & $2.3 \pm 0.2$ & $2.6 \pm 0.3$ \\
\hline Age at calving, years & $3.7 \pm 0.2$ & $4.6 \pm 0.4$ & $5.5 \pm 0.5$ \\
\hline Daily Milk Yield (Day 4 - Day 21), L & $28.6 \pm 0.5$ & $31.3 \pm 0.6$ & $30.9 \pm 0.7$ \\
\hline Day 4 - Day 6 & $23.0 \pm 0.9$ & $25.4 \pm 1.1$ & $27.4 \pm 0.9$ \\
\hline Day 7 - Day 9 & $26.6 \pm 1.0$ & $28.9 \pm 1.3$ & $31.3 \pm 1.7$ \\
\hline Day 10 - Day 12 & $28.8 \pm 1.1$ & $31.7 \pm 1.4$ & $30.8 \pm 1.8$ \\
\hline Day 13 - Day 15 & $30.4 \pm 1.1$ & $32.9 \pm 1.2$ & $32.2 \pm 1.7$ \\
\hline Day 16 - Day 18 & $30.6 \pm 1.1$ & $33.6 \pm 1.2$ & $31.2 \pm 1.7$ \\
\hline Day 19 - Day 21 & $31.8 \pm 1.1$ & $34.5 \pm 1.4$ & $33.0 \pm 1.9$ \\
\hline \multicolumn{4}{|c|}{ Incidence of ketosis by monitoring day } \\
\hline \multicolumn{4}{|l|}{ Day 0} \\
\hline Day 3 & & 3 & 2 \\
\hline Day 6 & & 6 & 5 \\
\hline Day 9 & & 8 & 10 \\
\hline Day 12 & & 2 & 3 \\
\hline Day 15 & & 5 & 5 \\
\hline Day 18 & & 7 & 4 \\
\hline Day 21 & & 3 & \\
\hline
\end{tabular}

\subsection{Association between ketosis and hematological parameters}

We analyzed whether hematological parameters on calving date were associated with ketosis. Significant differences in red blood cell (RBC) count, hemoglobin ( $\mathrm{Hb}), \mathrm{MCV}, \mathrm{MCH}, \mathrm{MCHC}$, RDW, and white blood cell (WBC), lymphocyte, monocyte, and eosinophil counts were found among the groups (Table 2). RBC, Hb, $\mathrm{MCHC}$, and lymphocyte counts were not associated with the severity of ketosis. However, the group with severe ketosis had significantly higher MCV and $\mathrm{MCH}$ and lower RDW and WBC, monocyte, and eosinophil counts; NK had the lowest MCV and $\mathrm{MCH}$, while CK had the highest values of MCV and $\mathrm{MCH}$. 
NK displayed the highest values of RDW and WBC, monocyte, and eosinophil counts, whereas CK exhibited the lowest values (Fig. 1). 
Table 2

The complete blood count (CBC) test results of each group. Abbreviation: RBC, red blood cell; $\mathrm{HCT}$, hematocrit; $\mathrm{Hb}$, Hemoglobin; MCV, mean corpuscular volume; $\mathrm{MCH}$, mean corpuscular hemoglobin; MCHC, mean corpuscular hemoglobin concentration; RDW, red cell distribution width; WBC, white blood cell; PLT, platelet; MPV, Mean platelet volume; PDW, Platelet distribution width; PCT, plateletcrit. $P$ values are expressed in 2 ways: mean ( $95 \%$ confidence interval), one-way ANOVA; mean \pm standard error of the means, Kruskal-Wallis test.

\begin{tabular}{|c|c|c|c|c|}
\hline & NK & SCK & CK & $p$ value \\
\hline \multicolumn{5}{|l|}{ Erythrocyte } \\
\hline \multirow[t]{2}{*}{ RBC count, $\mathrm{M} / \mu \mathrm{L}$} & 6.42 & 6.43 & 6.01 & 0.023 \\
\hline & $(6.27-6.59)$ & $(6.21-6.65)$ & $(5.67-6.35)$ & \\
\hline HCT, \% & $33.33 \pm 0.38$ & $34.69 \pm 0.56$ & $34.15 \pm 0.93$ & 0.076 \\
\hline $\mathrm{Hb}, \mathrm{g} / \mathrm{dL}$ & $11.07 \pm 0.12$ & $11.62 \pm 0.17$ & $11.15 \pm 0.27$ & 0.042 \\
\hline \multirow[t]{2}{*}{$M C V, f L$} & 52.09 & 54.19 & 56.94 & $<0.001$ \\
\hline & $(50.97-53.21)$ & $(52.46-55.92)$ & $(55.30-58.59)$ & \\
\hline \multirow[t]{2}{*}{$\mathrm{MCH}, \mathrm{pg}$} & 17.29 & 18.15 & 18.63 & $<0.001$ \\
\hline & $(17.00-17.59)$ & $(17.65-18.65)$ & $(18.23-19.04)$ & \\
\hline \multirow[t]{2}{*}{$\mathrm{MCHC}, \mathrm{g} / \mathrm{dL}$} & 33.24 & 33.62 & 32.76 & 0.003 \\
\hline & $(33.00-33.48)$ & $(33.31-33.94)$ & $(32.35-33.16)$ & \\
\hline \multirow[t]{2}{*}{ RDW, \% } & 25.71 & 25.20 & 23.78 & 0.002 \\
\hline & $(25.05-26.37)$ & $(24.40-26.00)$ & $(23.02-24.54)$ & \\
\hline \multirow[t]{2}{*}{ Reticulocyte, $\mathrm{K} / \mu \mathrm{L}$} & 1.60 & 2.00 & 1.94 & 0.339 \\
\hline & $(1.27-1.92)$ & $(1.42-2.58)$ & $(1.41-2.47)$ & \\
\hline \multicolumn{5}{|l|}{ Leukocyte } \\
\hline \multirow[t]{2}{*}{ WBC count, $\mathrm{K} / \mu \mathrm{L}$} & 13.20 & 12.29 & 10.37 & 0.004 \\
\hline & $(12.30-14.10)$ & $(11.13-13.44)$ & $(8.74-11.99)$ & \\
\hline Neutrophil, K/ $\mu \mathrm{L}$ & $3.23 \pm 0.44$ & $2.48 \pm 0.56$ & $2.76 \pm 0.57$ & 0.449 \\
\hline Lymphocyte, $\mathrm{K} / \mu \mathrm{L}$ & $7.55 \pm 0.49$ & $7.79 \pm 0.59$ & $5.94 \pm 0.76$ & 0.013 \\
\hline \multirow[t]{2}{*}{ Monocyte, $\mathrm{K} / \mu \mathrm{L}$} & 2.19 & 1.84 & 1.54 & $<0.001$ \\
\hline & $(2.00-2.37)$ & $(1.60-2.08)$ & $(1.32-1.76)$ & \\
\hline Eosinophil, K/ $\mu \mathrm{L}$ & $0.23 \pm 0.03$ & $0.17 \pm 0.02$ & $0.12 \pm 0.02$ & 0.009 \\
\hline Basophil, K/ $\mu \mathrm{L}$ & $0.005 \pm 0.001$ & $0.003 \pm 0.001$ & $0.004 \pm 0.001$ & 0.311 \\
\hline
\end{tabular}




\begin{tabular}{|lllll|}
\hline & NK & SCK & CK & p value \\
\hline Platelet & & & & \\
\hline PLT count, K/ $\mu \mathrm{L}$ & $329.24 \pm 11.38$ & $328.39 \pm 12.13$ & $302.18 \pm 14.87$ & 0.286 \\
\hline MPV, fL & $6.51 \pm 0.06$ & $6.75 \pm 0.09$ & $6.75 \pm 0.10$ & 0.051 \\
\hline PDW, fL & $7.84 \pm 0.12$ & $8.08 \pm 0.12$ & $8.00 \pm 0.15$ & 0.214 \\
\hline PCT, \% & $0.21 \pm 0.01$ & $0.22 \pm 0.01$ & $0.20 \pm 0.01$ & 0.250 \\
\hline
\end{tabular}

\subsection{Association between ketosis and serum biochemistry}

Serum biochemical and mineral parameters on calving date were analyzed to determine their association with ketosis. BHBA showed significant differences among groups on calving date despite small gap. There were significant differences in NEFA, total cholesterol (TC), TB, creatinine, alanine transaminase (ALT), lactate dehydrogenase (LDH), and amylase among groups. TC and creatinine levels failed to demonstrate a relationship with the severity of ketosis, but NEFA and TB had a positive relationship with the severity of ketosis. CK had the highest concentrations of NEFA and TB, while NK had the lowest concentrations of NEFA and TB. However, ALT, LDH, and amylase had negative relationships with the severity of ketosis; these parameters were higher in NK, SCK, and CK in order. ALB exhibited a negative relationship with the severity with weak significance $(p=0.051)$, and the NK group had a significantly elevated ALB value than the CK group (Table 3 and Fig. 2). 
Table 3

Serum biochemistry and mineral levels by each group. Abbreviation: BHBA, $\beta$ hydroxybutyrate; NEFA, non-esterified fatty acids; TG, triglyceride; TC, total cholesterol; TP, total protein; TB, total bilirubin; ALT, Alanine transaminase; AST, aspartate transaminase; ALP Alkaline, phosphatase; GGT, $\gamma$-glutamyl transferase; LDH, lactate dehydrogenase; BUN, blood urea nitrogen; $\mathrm{Mg}$, magnesium; $\mathrm{P}$, inorganic phosphorus. $P$ values are expressed in 2 ways: mean (95\% confidence interval), one-way ANOVA; mean \pm standard error of the means,

Kruskal-Wallis test.

\begin{tabular}{|c|c|c|c|c|}
\hline & NK & SCK & CK & $p$ value \\
\hline $\mathrm{BHBA}, \mathrm{mmol} / \mathrm{L}$ & $0.50 \pm 0.02$ & $0.62 \pm 0.04$ & $0.68 \pm 0.06$ & 0.009 \\
\hline Glucose, mg/dL & $68.37 \pm 1.58$ & $69.88 \pm 2.37$ & $73.79 \pm 3.65$ & 0.783 \\
\hline NEFA, uEq/L & $452.08 \pm 23.38$ & $621.85 \pm 53.28$ & $902.31 \pm 87.87$ & $<0.001$ \\
\hline TG, U/L & $3.87 \pm 0.25$ & $3.35 \pm 0.27$ & $2.93 \pm 0.16$ & 0.100 \\
\hline $\mathrm{TC}, \mathrm{mg} / \mathrm{dL}$ & $83.21 \pm 2.05$ & $83.26 \pm 3.19$ & $74.21 \pm 3.15$ & 0.037 \\
\hline $\mathrm{TP}, \mathrm{g} / \mathrm{dL}$ & $\begin{array}{l}6.74 \\
(6.52-6.96)\end{array}$ & $\begin{array}{l}6.83 \\
(6.55-7.11)\end{array}$ & $\begin{array}{l}6.85 \\
(6.58-7.13)\end{array}$ & 0.787 \\
\hline Albumin, $\mathrm{g} / \mathrm{dL}$ & $\begin{array}{l}3.84 \\
(3.74-3.93)\end{array}$ & $\begin{array}{l}3.74 \\
(3.65-3.83)\end{array}$ & $\begin{array}{l}3.66 \\
(3.52-3.79)\end{array}$ & 0.051 \\
\hline Globulin, g/dL & $\begin{array}{l}2.90 \\
(2.67-3.14)\end{array}$ & $\begin{array}{l}3.09 \\
(2.81-3.37)\end{array}$ & $\begin{array}{l}3.20 \\
(2.88-3.51)\end{array}$ & 0.277 \\
\hline $\mathrm{TB}, \mathrm{U} / \mathrm{L}$ & $0.39 \pm 0.02$ & $0.54 \pm 0.04$ & $0.70 \pm 0.07$ & $<0.001$ \\
\hline BUN, mg/dL & $\begin{array}{l}16.06 \\
(15.06-17.06)\end{array}$ & $\begin{array}{l}15.81 \\
(14.24-17.39)\end{array}$ & $\begin{array}{l}15.87 \\
(14.27-17.46)\end{array}$ & 0.955 \\
\hline Creatinine, $\mathrm{mg} / \mathrm{dL}$ & $\begin{array}{l}1.44 \\
(1.39-1.49)\end{array}$ & $\begin{array}{l}1.56 \\
(1.48-1.63)\end{array}$ & $\begin{array}{l}1.53 \\
(1.44-1.61)\end{array}$ & 0.031 \\
\hline$A L T, U / L$ & $\begin{array}{l}19.92 \\
(18.46-21.38)\end{array}$ & $\begin{array}{l}17.06 \\
(15.02-19.10)\end{array}$ & $\begin{array}{l}14.79 \\
(13.18-16.40)\end{array}$ & $<0.001$ \\
\hline AST, U/L & $86.21 \pm 2.66$ & $80.26 \pm 2.40$ & $81.62 \pm 5.42$ & 0.099 \\
\hline$A L P, U / L$ & $\begin{array}{l}230.30 \\
(209.62-250.97)\end{array}$ & $\begin{array}{l}207.06 \\
(178.33-235.79)\end{array}$ & $\begin{array}{l}205.10 \\
(177.76-232.44)\end{array}$ & 0.238 \\
\hline GGT, U/L & $\begin{array}{l}23.58 \\
(21.07-26.09)\end{array}$ & $\begin{array}{l}23.48 \\
(20.48-26.49)\end{array}$ & $\begin{array}{l}22.31 \\
(20.08-24.54)\end{array}$ & 0.804 \\
\hline $\mathrm{LDH}, \mathrm{U} / \mathrm{L}$ & $1030.44 \pm 19.34$ & $976.56 \pm 28.35$ & $926.79 \pm 26.84$ & 0.002 \\
\hline
\end{tabular}




\begin{tabular}{|lllll|}
\hline & NK & SCK & CK & $p$ value \\
\hline Amylase, U/L & 25.51 & 22.73 & 19.44 & 0.046 \\
& $(22.57-28.45)$ & $(19.40-26.07)$ & $(15.36-23.53)$ & \\
\hline Lipase, U/L & $83.17 \pm 6.84$ & $70.16 \pm 5.62$ & $87.04 \pm 13.28$ & 0.880 \\
\hline Calcium, mg/dL & $8.10 \pm 0.14$ & $7.83 \pm 0.27$ & $7.86 \pm 0.24$ & 0.449 \\
\hline Mg, mg/dL & $2.33 \pm 0.05$ & $2.49 \pm 0.07$ & $2.53 \pm 0.10$ & 0.092 \\
\hline P, mg/dL & $5.09 \pm 0.12$ & $5.02 \pm 0.29$ & $4.62 \pm 0.29$ & 0.187 \\
\hline
\end{tabular}

\section{Discussion}

The present study aimed to predict whether Holstein Friesian cows are at risk of ketosis during the transition period by analyzing hematological and serum biochemical parameters on the calving date. To the best of our knowledge, this study is the first to identify the associations of hematological and serum biochemical parameters on calving date with ketosis during the postpartum transition period. We observed that elevated MCV, MCH, NEFA and TB were positively associated with ketosis and its severity. The cows with greater RDW, WBC count, monocyte count, eosinophil count, ALB, ALT, LDH, and amylase were at lower risk of ketosis and its severity. MCV, MCHC, lymphocyte count, and eosinophil count were significantly different between subclinical and clinical ketosis.

Red blood cell indices are associated with body dysfunction. However, only a few previous studies investigated the association between ketosis and red blood cell indices in cows, and they showed no association between ketosis and $\mathrm{MCV}, \mathrm{MCH}, \mathrm{MCHC}$, and $\mathrm{RDW}{ }^{13,22}$; the finding is different from that in the current study. As shown in Table 1, the incidence of ketosis varied during the transition period. The discrepancy between our findings and the previous findings may stem from differences in study design, such as monitoring frequency. The liver is an organ closely related to ketosis. Humans with liver disease and deficiencies in vitamin $\mathrm{B}_{9}$ or $\mathrm{B}_{12}$ showed elevated $\mathrm{MCV}$ and $\mathrm{MCH}^{23,24}$. Dairy cows with elevated MCV and $\mathrm{MCH}$ have a weak liver, which may lead to ketosis. RDW is known to be elevated in patients with liver disease. Interestingly, the RDW findings in this study are inconsistent with those in previous studies on liver diseases; the reason is unclear, but a previous study showed a similar association between RDW and the severity of ketosis in sheep, although it was not significant ${ }^{25}$. Our findings suggest that unlike cows with other liver diseases, those with severer ketosis have lower RDW values.

Accumulating studies indicate that ketosis is associated with decreased WBC count, and ketosis increases the risk of diseases. Dairy cows with ketosis show decreased WBC count ${ }^{14,26}$. Human adults with ketogenic diets show a significant decrease in WBC count ${ }^{27}$. Ketosis increases the risk of metritis and mastitis ${ }^{28}$. Consistently, the present study found lower WBC counts and severer ketosis during the transition period. The decrease in WBC count in cows with ketosis might contribute to the susceptibility to 
infectious diseases. NK cows had extremely significantly more monocytes than CK cows and significantly more monocytes than SCK cows. Both cows and humans with hyperketonemia have lower monocyte counts ${ }^{29,30}$. Humans in the low-energy state have a reduced number of circulating monocytes migrated from bone marrow, by signaling from hepatocyte ${ }^{31}$. The liver might already play a role in recruiting a smaller population of monocytes from the bone marrow before ketosis in the ketosis groups. In addition, NK cows showed very significantly more eosinophils than SCK cows, while SCK cows had significantly more eosinophils than CK cows. Human with ketosis also showed a lower population of eosinophil ${ }^{32,33}$. It is speculated that eosinophil count is lowered by oxidative stress, elevated apoptosis, and the inhibition of cell proliferation ${ }^{32}$.

As for serum biochemistry, most of the parameters associated with ketosis (NEFA, ALB, TB, ALT, and amylase) are highly related to hepatic dysfunction. A negative energy balance in animals promotes lipolysis, which mobilizes NEFA from the adipose tissue to most body tissues through the blood stream. The liver is the main organ to process NEFA into 3 forms: ATP via complete oxidation, ketone bodies, or very low-density lipoproteins via incomplete oxidation ${ }^{4,34}$. The liver of ketosis cows may not be able to remove NEFA compared to non-ketosis cows around the parturition before ketosis onset. In addition, ALB and TB are commonly used to indicate damage to the liver, and our results on ALB and TB correspond with previous findings. CK, SCK, and NK had lower serum ALB levels in order, which indicate from hepatic dysfunction. ${ }^{21,35}$. TB increases in ketosis, indicating liver damage ${ }^{18,36}$, and a higher level in TB in parturition suggests hepatic dysfunction. Notably, the ALT results in the current study are different from those in previous studies, which demonstrated that ALT is insensitive in ruminants and cows with ALT levels $21,37,38$. The difference might be due to the study design. Hepatic apoptosis is elevated in dairy cows with ketosis ${ }^{39}$. Vaden et al. showed that low levels of ALT indicate hepatic atrophy, while high levels of ALT indicate hepatocellular injury or leakage ${ }^{40}$. CK cows had the lowest level of ALT while NK cows had the highest on the parturition day. The findings suggest that the extent of hepatic atrophy in Holstein cattle is linked to ketosis.

To the best of our knowledge, no study has reported an association between serum amylase and ketosis in cattle. However, low concentrations of serum amylase are associated with the degree of liver dysfunction, such as non-alcoholic fatty liver, metabolic syndrome, and insulin resistance ${ }^{41,42}$. In human medicine, a previous study revealed that low serum amylase levels were inversely related to serum ketone body ${ }^{43}$. Insulin resistance is also related to ketosis in dairy cows ${ }^{44}$. Our findings suggest that ketosis may be associated with low serum amylase concentration.

LDH is known to contribute glycolysis, and the elevated levels of LDH are a marker of organ damage, including muscles and liver damage ${ }^{45}$. LDH was expected to be higher in cows with ketosis because other biomarkers indicated obvious damage to the liver. However, we found that LDH had a negative relationship with the severity of ketosis. Information on the association between LDH and ketosis is limited. Energy production might presumably be related to our unexpected findings that NK had the highest level of LDH and CK had the lowest level of LDH. Indeed, LDH plays an important role in the 
regulation of glycolysis ${ }^{46}$. Lower level of LDH in ketosis might indicate a decreased ability to use glucose for energy, which might lead to fat mobilization.

The limitation of our study is that it was conducted at only one farm. However, the current study demonstrated that the parameters of the complete blood count (CBC) test and serum biochemistry on the calving date were associated with ketosis and its severity. The parameters were statistically significantly different based on the severity of ketosis. Our findings suggest that theses parameters are predictive indicators for ketosis.

In summary, the present study found that hematological and serum biochemical parameters on the calving date were associated with ketosis and its severity during the postpartum transition period. Dairy Holstein cows with clinical ketosis showed significantly the highest values of MCV, MCH, NEFA, and TB but significantly lowest values of RDW; WBC, monocyte, and eosinophil counts; and ALB, ALT, LDH, and amylase levels. Dairy Holstein cows with non-ketosis showed the opposite results. Our findings suggest that dairy Holstein cows, that are susceptible to ketosis, can be selected by hematological and serum biochemical parameters.

\section{Materials And Methods}

\subsection{Animals}

A total of 126 Holstein Friesian cows were used in this study. The cows were raised and calved at one farm of National Institute of Animal Science, in Cheonan, Republic of Korea. The animals calved from January 2018 to January 2021 and were normally milked during the transition period. The animals were fed the same total mixed rations ad libitum, which were composed of concentrates, soybean meal, corn silage, alfalfa hay, timothy hay, enzyme, minerals, and vitamin additives. They were milked twice a day in the morning and evening. Dairy cows that were milked less than twice a day or were offered any medication or nutritional or microbial supplement capsules and boluses after calving were excluded.

\subsection{Blood sampling and case definition}

Blood sampling was conducted once every three days (8 times in 21 days from the calving date) during the postpartum transition period. Blood was sampled once from the jugular vein of the cows in 6-23 hours postpartum on the calving date and seven times in the morning from day 3 postpartum when the cows began to be fed as soon as they finished milking. Blood was collected using ethylenediaminetetraacetic acid (EDTA) and serum-separating tube (SST) tubes. BHBA was measured with electronic handheld meters (FreeStyle Optimum Neo, Abbott Diabetes Care Ltd., Witney, UK) and $\beta$ ketone test strips (FreeStyle Optimum H $\beta$-ketone, Abbott Diabetes Care Ltd., Witney, UK) immediately after blood sampling.

The cows were divided into three groups according to the highest BHBA concentration in the eight samples during the post-calving transition period: a non-ketosis group $(<1.2 \mathrm{mmol} / \mathrm{l}, \mathrm{n}=63)$, a subclinical 
ketosis group (1.2 $\leq$ BHBA $\leq 2.9 \mathrm{mmol} / \mathrm{l}, \mathrm{n}=34)$, a clinical ketosis group $(\geq 3.0 \mathrm{mmol} / \mathrm{l}, \mathrm{n}=29){ }^{10,47}$.

\subsection{Blood analysis and Data collection}

The $\mathrm{CBC}$ test was performed with a hematology analyzer (Procyte Dx® hematology analyzer, IDEXX Laboratories, Westbrook, MA, USA) with blood collected in EDTA tubes. The profiles of the CBC test consisted of three types: erythrocytes, leukocytes, and platelets. The erythrocyte parameters included RBC count, hematocrit (HCT), Hb, MCV, MCH, MCHC, RDW, and reticulocyte count. The leukocyte parameters included WBC, neutrophil, lymphocyte, monocyte, eosinophil, and basophil counts. The platelet parameters included platelet (PLT) count, mean platelet volume (MPV), platelet distribution width (PDW), and plateletcrit (PCT).

Serum was harvested by centrifuging SST tubes at 3,000 rpm for 10 minutes. Serum biochemistry and mineral analyses were conducted using two biochemistry automatic analyzers (Catalyst ${ }^{\text {TM }}$ Dx chemistry analyzer, IDEXX Laboratories, Westbrook, MA, USA and Hitachi 7180, Hitachi Ltd., Tokyo, Japan). TB, amylase, and lipase were analyzed immediately after the serum was harvested using a Catalyst ${ }^{\mathrm{TM}} \mathrm{Dx}$ chemistry analyzer. Glucose, NEFA, TG, TC, total protein (TP), ALB, BUN, creatinine, ALT, aspartate transaminase (AST), alkaline phosphatase (ALP), gamma-glutamyl transferase (GGT), LDH, calcium, magnesium, and inorganic phosphorus were measured using a Hitachi 7180 with commercial enzyme assay kits from Wako (Fujifilm Wako Pure Chemical Ltd., Osaka, Japan). Globulin was calculated by subtracting ALB from TP. The serum was frozen and stored at $-70{ }^{\circ} \mathrm{C}$ pending analysis using a Hitachi 7180 .

\subsection{Statistical analyses}

Statistical analyses were performed using SPSS software (version 26.0; IBM Corp., Armonk, NY, USA). The Shapiro-Wilk test and Levene's test were used for normality analysis and equality of variances for oneway analysis of variance (ANOVA). The Kruskal-Wallis test was used for the parameters that did not satisfy normality analysis nor equality of variances. The difference analysis among groups was conducted with post-hoc Tukey honestly significant difference for One-way ANOVA and Mann-Whitney U test with Bonferroni's method for the Kruskal-Wallis test. One-way ANOVA was used to evaluate RBC count, MCV, MCH, MCHC, RDW, reticulocyte count, WBC count, Monocyte count, TP, ALB, globulin, BUN, creatinine, ALT, ALP, GGT, and amylase. The Kruskal-Wallis test was applied to analyze HCT, Hb, neutrophil count, lymphocyte count, eosinophil count, basophil count, PLT count, MPV, PDW, PCT, BHBA, glucose, NEFA, TG, TC, TB, AST, LDH, lipase, Ca, Mg, and P. Data obtained using one-way ANOVA are expressed as mean ( $95 \%$ confidence interval), whereas data obtained using the Kruskal-Wallis test are expressed as mean \pm standard error of the means.

Significance levels were interpreted based on $P$-values as follows: $0.01 \leq p<0.05$, significant; $0.001 \leq p<$ 0.01 , very significant; and $p<0.001$, extremely significant. Significance levels using Mann-Whitney U test with Bonferroni's test were divided into the following three categories: $0.003 \leq p<0.017$, significant; $0.0003 \leq p<0.003$, very significant; and $p<0.0003$, extremely significant. 


\subsection{Ethical approval}

This research was approved by the Institutional Animal Care and Use Committee (IACUC) at the National Institute of Animal Science, the Republic of Korea (approved number: NIAS-2020127). All experimental procedures involving animals were conducted in strict accordance with relevant guidelines and regulations.

\section{Declarations}

\section{Competing interests}

The authors declare no competing interests.

\section{Acknowledgements}

This work was supported by "The improvement of animal diseases control in National Institute of Animal Science (Project No. PJ01567603)" project at National Institute of Animal Science, Rural Development Administration, Republic of Korea. Furthermore, the authors would like to express our deepest gratitude to Youngso Hong, Hyunhoon Sung, and Jihyeon Lee for their technical support.

\section{Contributions}

S.M.H. and J.H.P. conceptualized and designed the study; S.M.H., S.-I.O., and S.H.K. analyzed the data; S.M.H., S.J.K., M.H.H., H.J.C., and J.H.P. interpreted the results; S.M.H. and J.H.L prepared the figures. S.M.H. and J.H.P. drafted the manuscript; All authors have approved the final version of the manuscript.

\section{Competing interests}

The authors declare no competing interests.

\section{Data availability}

The dataset analyzed for the current study is available from the authors upon reasonable request.

\section{References}

\section{Figures}



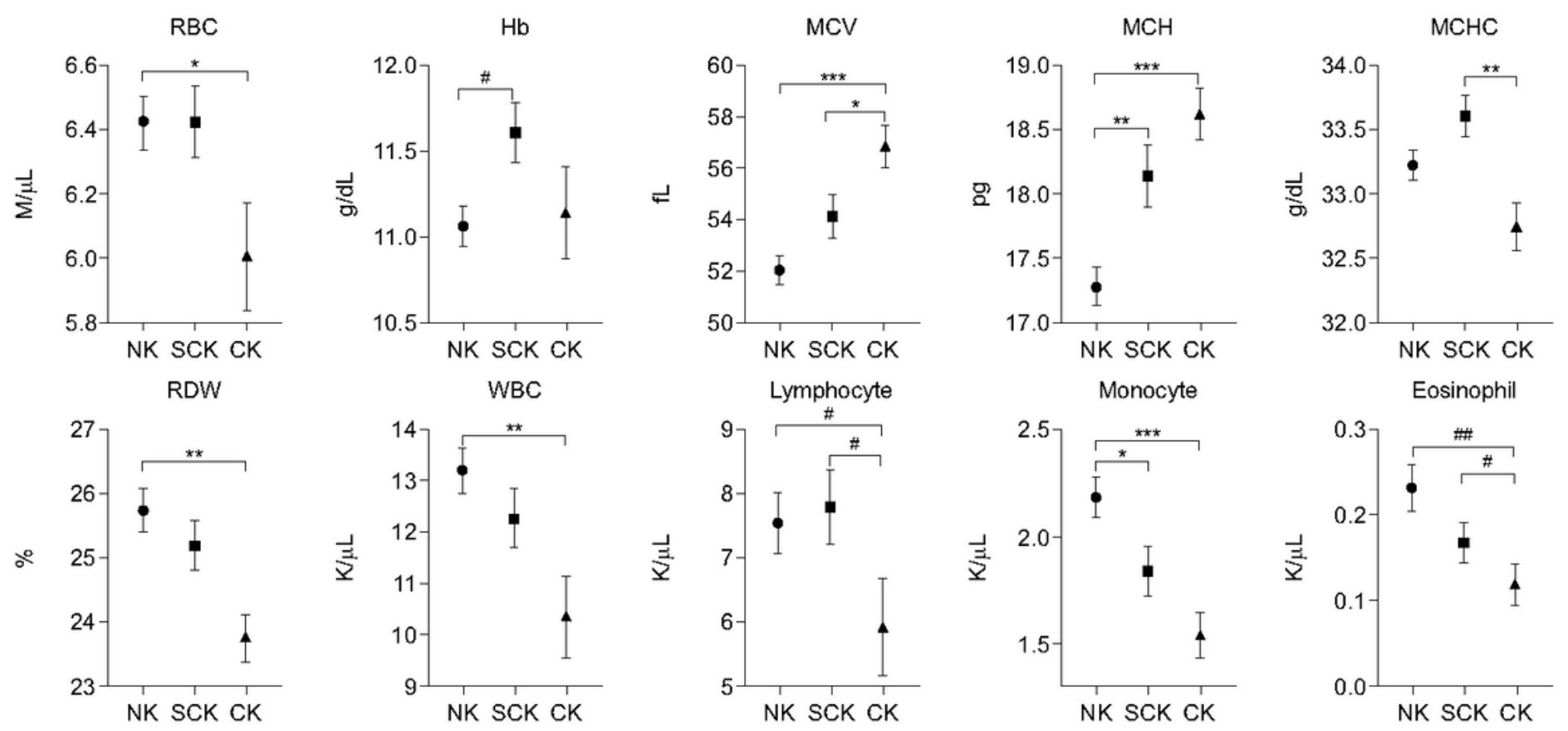

\section{Figure 1}

Hematological parameters in erythrocyte and leukocyte with statistical significance. The results are expressed as mean \pm standard error of the means. Statistical significance was expressed in 2 ways: oneway ANOVA with post-hoc Tukey honestly significant difference, ${ }^{*} \mathrm{P}<0.05 ;{ }^{*} \mathrm{P}<0.01$; ${ }^{\star \star \star} \mathrm{P}<0.001$. Kruskal-Wallis test and Mann-Whitney U test with Bonferroni's method, \#P $<0.017$; \#\#P $<0.003$.
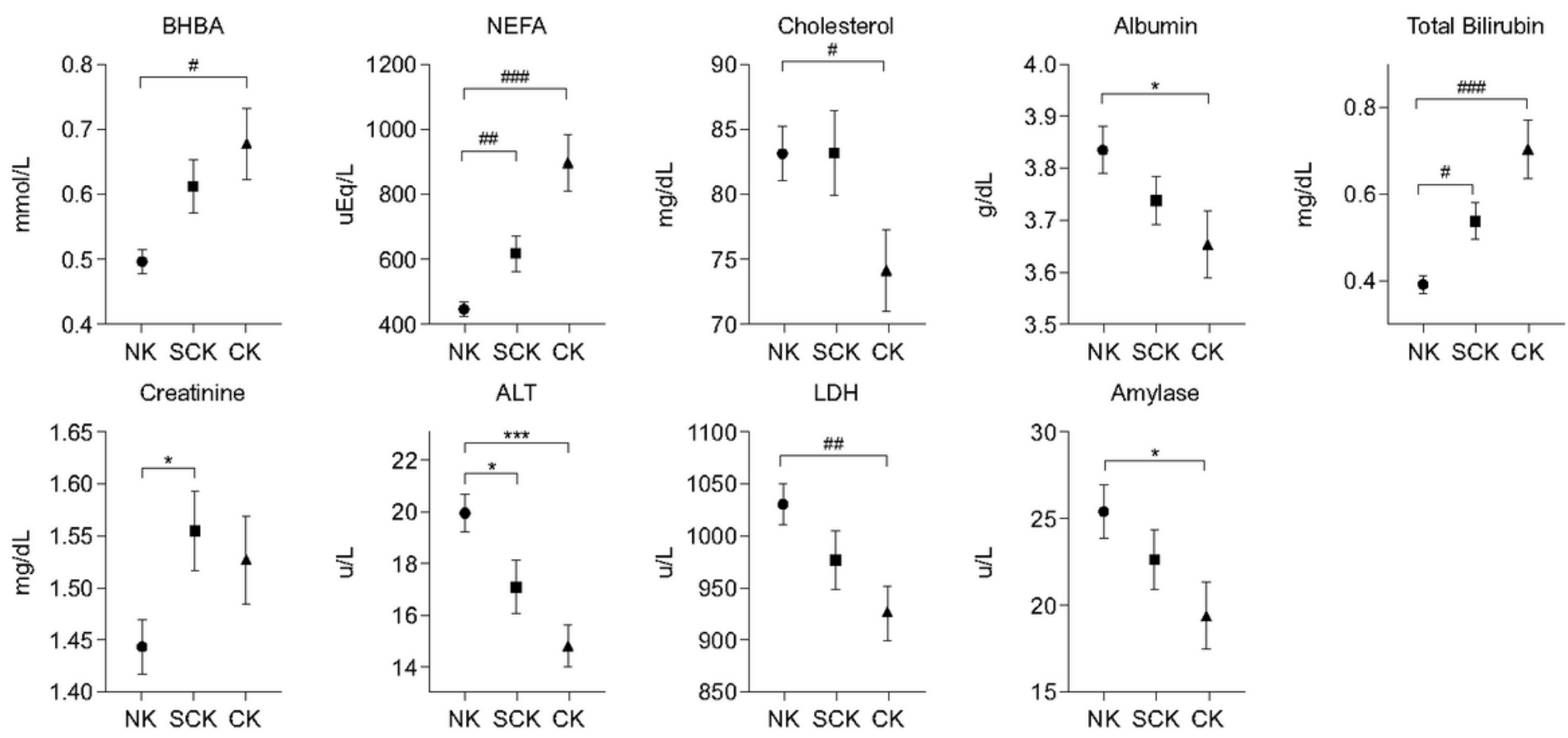

Figure 2 
Serum biochemical parameters with statistical significance. The results are expressed as mean \pm standard error of the means. Statistical significance was expressed in 2 ways: one-way ANOVA with posthoc Tukey honestly significant difference, ${ }^{\star} \mathrm{P}<0.05$; ${ }^{\star *} \mathrm{P}<0.01$; $* \star \star \mathrm{P}<0.001$. Kruskal-Wallis test and Mann-Whitney U test with Bonferroni's method, \#P<0.017; \#\#P $<0.003$, \#\#P $<0.0003$. 